\title{
Gut microbiota characteristics in mice with antibiotic-associated diarrhea
}

Haoqing Shao ${ }^{1,2}$, Chenyang Zhang ${ }^{1,2}$, Nenqun $X_{i a o^{3}}$ and Zhoujin $\operatorname{Tan}^{2,4^{*}}$ (D)

\begin{abstract}
Background: Antibiotic-associated diarrhea (AAD), defined as diarrhea that occurs in association with the administration of antibiotics and without another clear etiology, is one of the most common adverse drug events of antibiotics therapy. We established a diarrhea model induced by gentamycin and cefradine to investigate the microbiota characteristics in the intestinal lumen of mice with AAD and provide insights into noteworthy bacteria related to gentamicin and cefradine-associated diarrhea.

Results: The number of OTUs in the model group and the normal group was 983 and 2107, respectively, and 872 identical OTUs were shared between two groups. Species richness and species diversity of intestinal microbe were altered by antibiotics administration. PCOA showed a clear separation between AAD and health control. The dominant phyla of AAD mice were Firmicutes (52.63\%) and Proteobacteria (46.37\%). Among the genus with top 20 abundance, the relative abundance of 7 genera, Ruminococcus, Blautia, Enterococcus, Eubacterium, Clostridium, Coprococcus, and Aerococcus, were enriched in the model group. Based upon the LEfSe analysis, Enterococcus, Eubacterium, Ruminococcus, and Blautia were identified as potential biomarkers for AAD.

Conclusions: The bacterial diversity of the intestinal lumen was diminished after gentamicin and cefradine administration. The alterations in the abundance and composition of gut microbiota further led to the dysfunction of gut microbiota. More specifically, gentamicin and cefradine significantly increased the abundance of the opportunistic pathogens, of which Enterococcus and Clostridium were the most prominent and most worthy of attention.
\end{abstract}

Keywords: Gut microbiota, Antibiotic-associated diarrhea, Gentamicin, Cefradine, Enterococcus, Clostridium, $16 \mathrm{~S}$ rRNA gene sequencing

\section{Background}

Antibiotics are frequently prescribed drugs for clinical treatment of various bacterial infections. Yet several adverse drug events (ADE) have emerged as the widespread use of antibiotics. Antibiotic-associated diarrhea (AAD), defined as diarrhea that occurs in association with the administration of antibiotics and without

\footnotetext{
* Correspondence: tanzhjin@sohu.com

${ }^{2}$ Hunan Key Laboratory of TCM Prescription and Syndromes Translational Medicine, Changsha, Hunan, China

${ }^{4}$ School of Medicine, Hunan University of Chinese Medicine, Changsha, Hunan, China

Full list of author information is available at the end of the article
}

another clear etiology, is one of the most common $\mathrm{ADE}$ of antibiotics administration [1].

AAD is a complex disease that is affected by the host, infectious agent involved, and numerous clinical elements, including antibiotics therapeutic scheme. AAD can affect up to a third of the patients receiving a particular antibiotic [2], but the incidence and severity of AAD vary among different antibiotics. We observed more serious diarrhea in mice which received cephradine + gentamycin sulfate than in mice which received lincomycin hydrochloride + ampicillin sodium and ceftriaxone sodium + erythromycin lactobionate [3]. Numerous studies reported that the mechanisms for AAD 
mainly laid on the changes or dysbiosis of microbial composition and function induced by antibiotics [4-7]. Although recent studies were concentrated on $C$. difficile-associated diarrhea, it accounts for only $10-25 \%$ of all AAD cases [7]. The etiological factor in the majority of AAD cases remains undetermined. Bacteria such as Clostridium perfringens, Staphylococcus aureus, and Klebsiella oxytoca were also related to AAD [8]. Understanding the different etiological factors and pathogenesis that implicated in AAD may help to prevent AAD and reduce costs.

Gut microbiota is the trillions of microorganisms that normally inhabit the intestine of humans and other animals [9]. It is currently considered to be a highly complex ecosystem in which there are tremendous interdependencies and interactions between microbial species and between the microbes and their host [5]. The gene pool of the microbial inhabitants is considerably diverse and almost 100 times larger than the gene pool of the host [10]. Gut microbes contribute functional genes and metabolites which affect host metabolism, immune, endocrine, and other physiological processes and are, therefore, increasingly recognized as a necessary and key factor for maintaining health [11]. The microbial composition of the gut microbiota can be influenced by lifestyle, diet as well as drugs and varied across the digestive tract. The colon, containing a densely-populated microbial ecosystem with up to $10^{12}$ cells per gram of intestinal content, harbors much greater microbial abundance and diversity relative to the small intestine [12]. Consequently, microbial analyses of intestinal content or stool have been wildly adopted as measures to surmise the role of gut microbe in host health and disease, based on the belief that it represents all microbial populations throughout the intestines [12]. Numerous studies have confirmed that disruptions in the composition and function of the gut microbe are associated with diseases ranging from localized gastrointestinal disorders to neurological, respiratory, metabolic, hepatic, and cardiovascular illnesses [13-15]. Many efforts are currently focused on exploring potential causality and related microbe-mediated disease mechanisms, with the hope that an improved understanding will fuel the conception and realization of novel therapeutic and preventive strategies [16]. There is increasing evidence for the effectiveness of probiotics, particularly Lactobacillus rhamnosus or Saccharomyces boulardii, and fecal microbiota transplantation in preventing or treating $\operatorname{AAD}[17,18]$. Advances in our understanding of microbiota characteristics might set the foundation for designing therapies that target the gut microbiota to prevent and treat AAD.

In the previous study, we found that a combination of gentamycin and cefradine showed a higher diarrhea incidence than a combination of lincomycin and ampicillin and a combination of ceftriaxone and erythromycin [3]. To further investigate potential pathogens responding to gentamicin and cefradine-associated diarrhea and better understand the different mechanisms of AAD, we analyzed the characteristics of gut microbiota in AAD model mice and healthy individuals.

\section{Results}

\section{Analysis of $16 \mathrm{~S}$ rRNA gene sequences and operational taxonomic unit}

A total of 766,407 high-quality sequences were detected from 6 samples belonging to two groups, with a length distribution concentrated at $225 \mathrm{bp}$. The Good's coverages calculated by Mothur to reflect the depth of sequencing were greater than $99.75 \%$ (from 0.9975 to 0.9997), which indicates that the sequencing results represent the true condition of the microorganisms in the sample. Finally, these high-quality sequences were clustered into 2218 OTUs (after quality-filtering) based on similarity at a $97 \%$ threshold. The number of OTUs in groups control and model was 2107 and 983, respectively (Fig. 1). There were 872 identical OTUs between the two groups, and the percentage of identical OTUs was $39.31 \%$.

\section{Diversity analysis of intestinal microbiota}

Alpha diversity indexes are regularly adopted in ecology to estimate the richness of microbial species and quantitatively describe microbial species diversity in a community. The greater the Chaol and ACE index, the richer the species in a community. The larger the Shannon

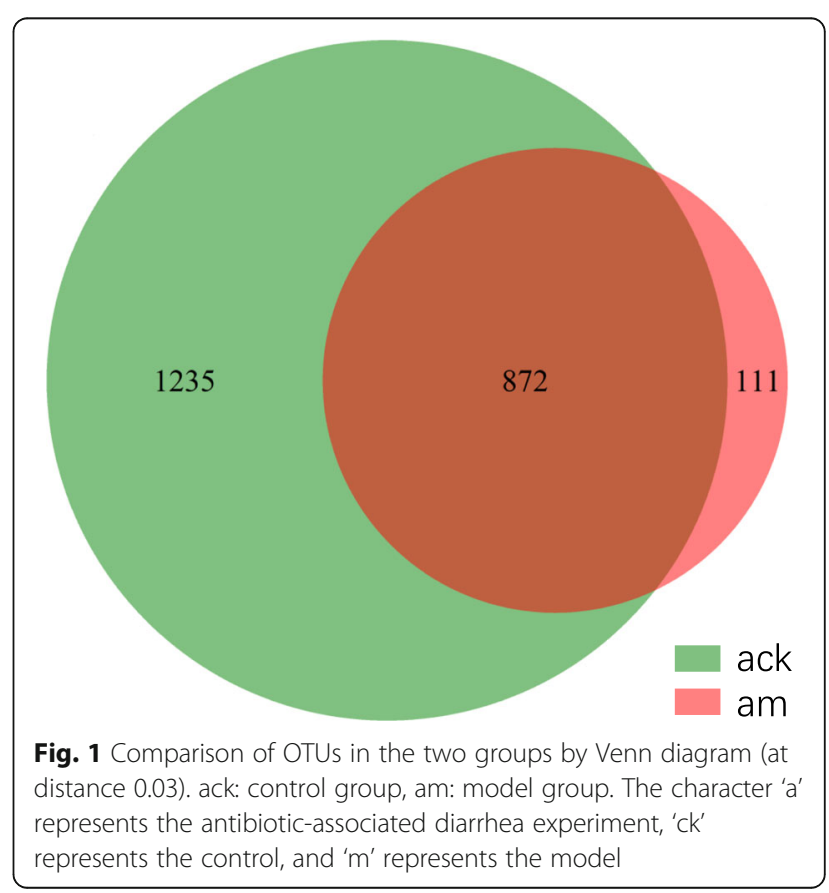


value and the smaller the Simpson value, the higher the diversity in a community. In this study, the Chao1, ACE, Simpson, and Shannon index in the model group were $672.28 \pm 227.51,664.43 \pm 232.91,0.33 \pm 0.07$, and $2.06 \pm$ 0.17 , which were lower than $1049.31 \pm 828.04,1035.84 \pm$ $825.48,0.34 \pm 0.39$, and $2.83 \pm 1.97$ in the control group (Fig. 2a).

Beta diversity measures the change in the diversity of species from one environment to another. The principal coordinates analysis (PCoA) based on unweighted UniFrac distances (accounting for the abundance of OTUs) was performed to explore and to visualize the dissimilarities of microbial communities between two groups (Fig. $2 \mathrm{~b})$. The results showed that, on the whole, the distance between groups was further than that within groups meaning that the microbial communities were different between the model group and normal group.

\section{Comparison of gut microbiota at the phylum level}

After OTUs less than $0.001 \%$ were filtered, the remaining OTUs were classified into 38 phyla, 115 classes, 223 orders, 347 families, and 516 genera (Fig. 3). Overall, the dominant phyla of the gut microbiome in mice were changed from Firmicutes, Bacteroidetes, and Proteobacteria to Firmicutes and Proteobacteria after antibiotics administration. Compared to the control group, the model group had an over-representation of Proteobacteria $(13.07 \%$ vs $46.37 \%)$, and lower abundances of Firmicutes $(63.52 \%$ vs $52.63 \%)$, Bacteroidetes $(17.27 \%$ vs $0.29 \%)$, Actinobacteria (1.14\% vs $0.25 \%$ ) and Planctomycetes $(1.32 \%$ vs $0.09 \%)$ (Fig. 4$)$. There was a significantly higher Firmicutes to Bacteroidetes $(\mathrm{F} / \mathrm{B})$ ratio in samples of AAD model group $(p \mathrm{FDR}<0.05)$.

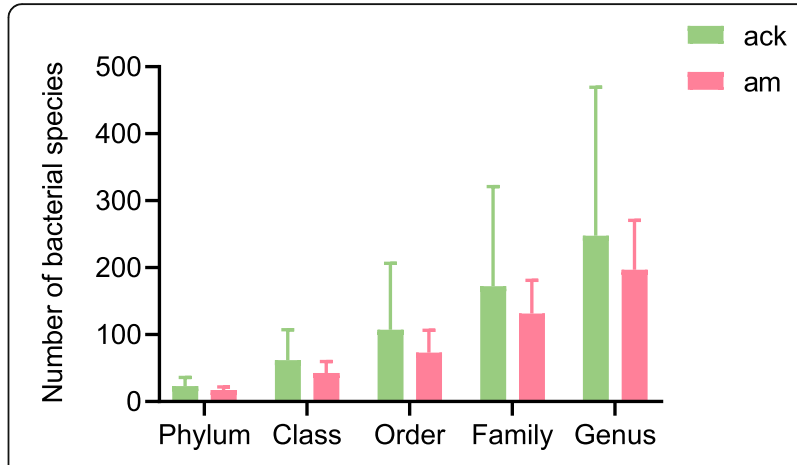

Fig. 3 The number of microbial species of each sample at different taxonomic levels. Data are $\bar{x} \pm \mathrm{SD}, n=3, p>0.05$ (Mann-Whitney $\mathrm{U}$ test). ack: control group, am: model group

\section{Antibiotics changed the dominant bacteria of intestinal} contents at the genus level

A phylogenetic dendrogram was performed to reflect the information of species and abundance of mice gut microbiome (Fig. 5). Bacillus, Lactobacillus, Ruminococcus, Enterococcus, Blautia, and Eubacterium were especially showed in color based on their abundance of over $1 \%$. The top 20 genera in abundance were shown in a heat map (Fig. 6). Among the genus with the top 20 abundance, the relative abundance of 7 genera, Ruminococcus, Blautia, Enterococcus, Eubacterium, Clostridium, Coprococcus, and Aerococcus, were enriched in the model group. Moreover, genera with abundance over 1\% in group model were Lactobacillus (23.21\%), Enterococcus (5.97\%), Blautia (5.46\%), Ruminococcus (5.37\%), Bacillus (5.24\%), Eubacterium (4.62\%), Pseudomonas (2.09\%) and Clostridium (1.79\%). However, there are
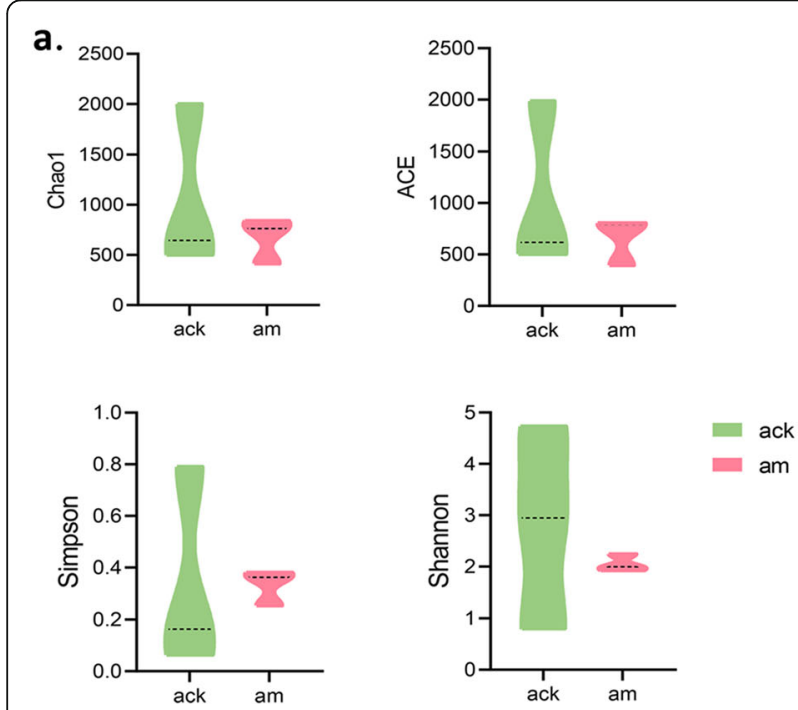

b.

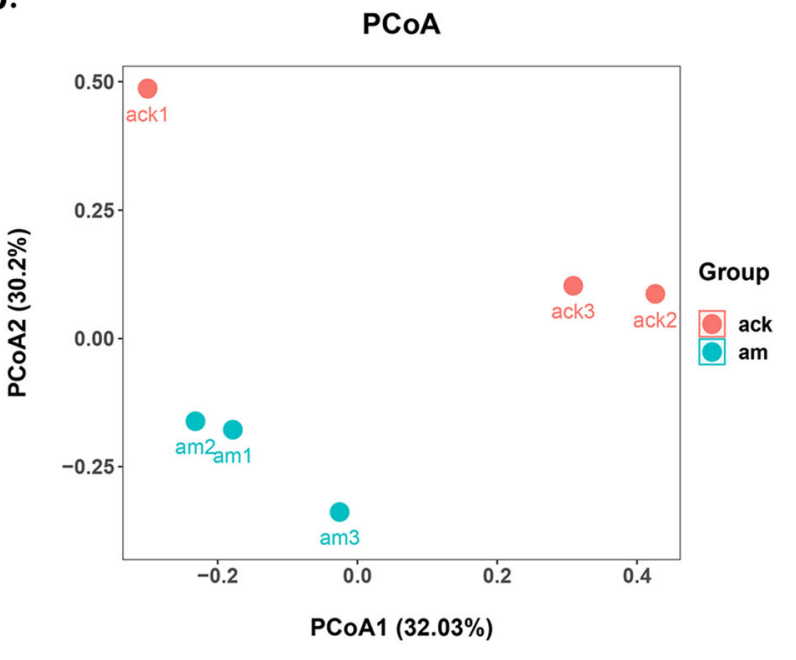

Fig. 2 Diversity analysis of gut microbiota. a Grouping histogram of Alpha diversity indexes. Data are $\bar{x} \pm \mathrm{SD}, n=3, p>0.05$ (Mann-Whitney $U$ test). b PCoA plot based on unweighted UniFrac distances. Ack: control group, am: model group 


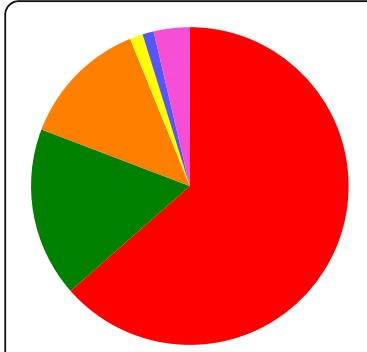

Group ack
- $63.52 \% 222700$ Firmicutes

- $17.27 \% 60554$ Bacteroidetes

- $13.07 \% \quad 45837$ Proteobacteria

- $1.32 \% 4634$ Planctomycetes

- $1.14 \% 4014$ Actinobacteria

- $3.66 \% 12845$ Others

Total sequence number $=350584$

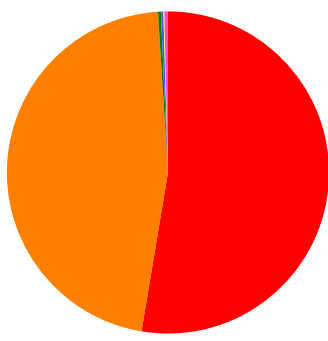

Group am
- $52.63 \% 213670$ Firmicutes

- $46.37 \% 188261$ Proteobacteria

- $0.29 \% 1159$ Bacteroidetes

- $0.25 \% 1026$ Actinobacteria

- $0.09 \% 384$ Planctomycetes

- $0.36 \% 1469$ Others

Total sequence number $=405969$

Fig. 4 Gut microbiota structure at the phylum level. Ack: control group, am: model group

only 2 genera with an abundance of over $1 \%$ in group control, which is Bacillus (40.46\%) and Lactobacillus (13.43\%).

\section{Taxa with significantly different abundances between groups by LEfSe}

To determine the variations in gut microbiota composition between the normal and AAD model mice, linear discriminant analysis effect size (LEfSe) was performed to find which taxa were enriched in the two groups
(Fig. 7). LEfSe emphasizes both statistical significance and biological relevance, allowing researchers to identify differentially abundant features that are also consistent with biologically meaningful categories (subclasses) [19]. Although LEfSe revealed that the genera Enterococcus, Eubacterium, Blautia, and Ruminococcus were the most abundant in the model group, this dominance of the latter two was not observed in all three samples of the model group (Fig. 7). Relatively increased numbers of Enterococcus and Eubacterium were observed in all three samples of model group, indicating that the two genera

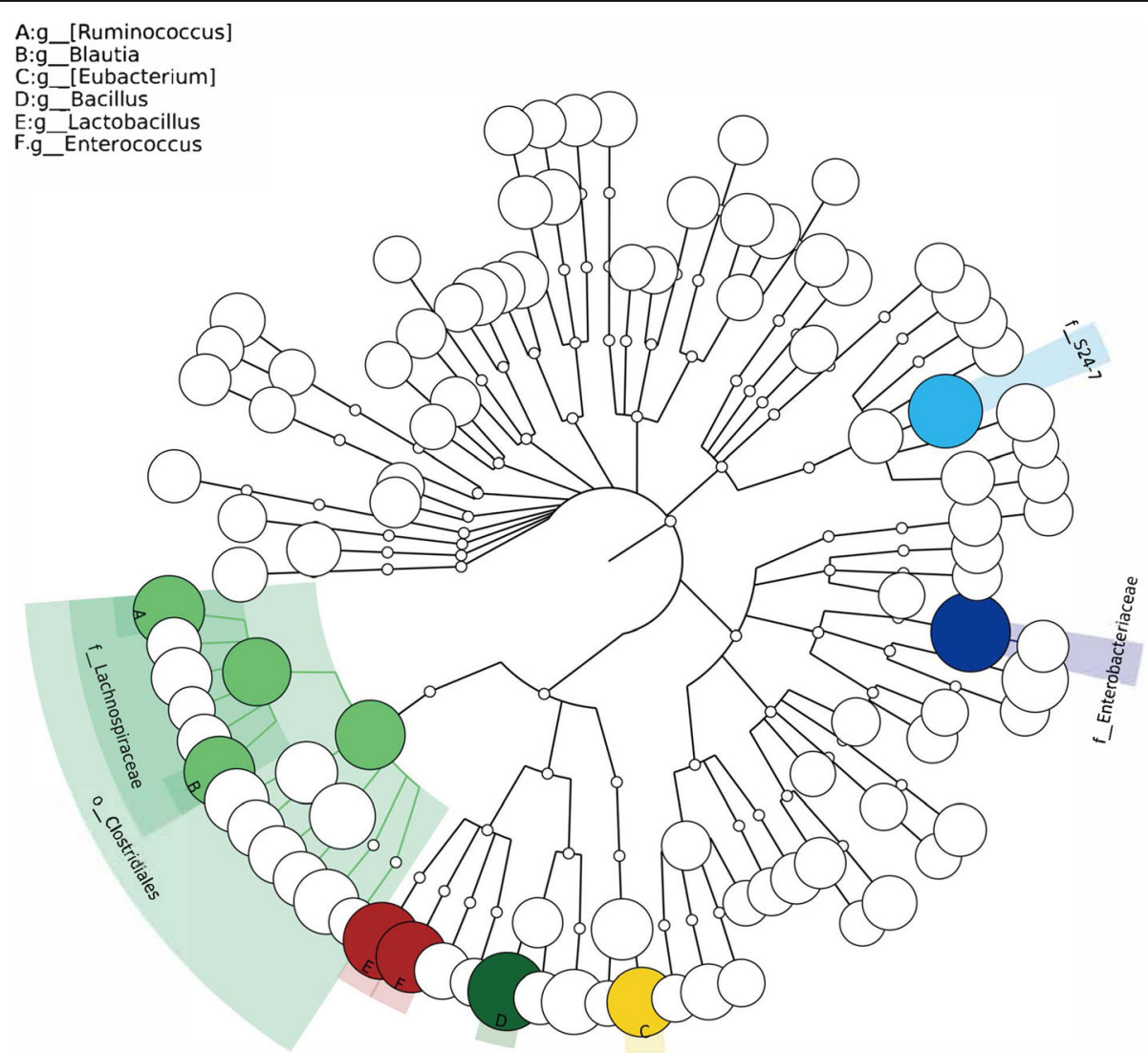

Fig. 5 Phylogenetic dendrogram analysis based on species and abundance of mice gut microbe at the genus level. The node size represents the average relative abundance, and species with abundance $>1 \%$ were shown in color 


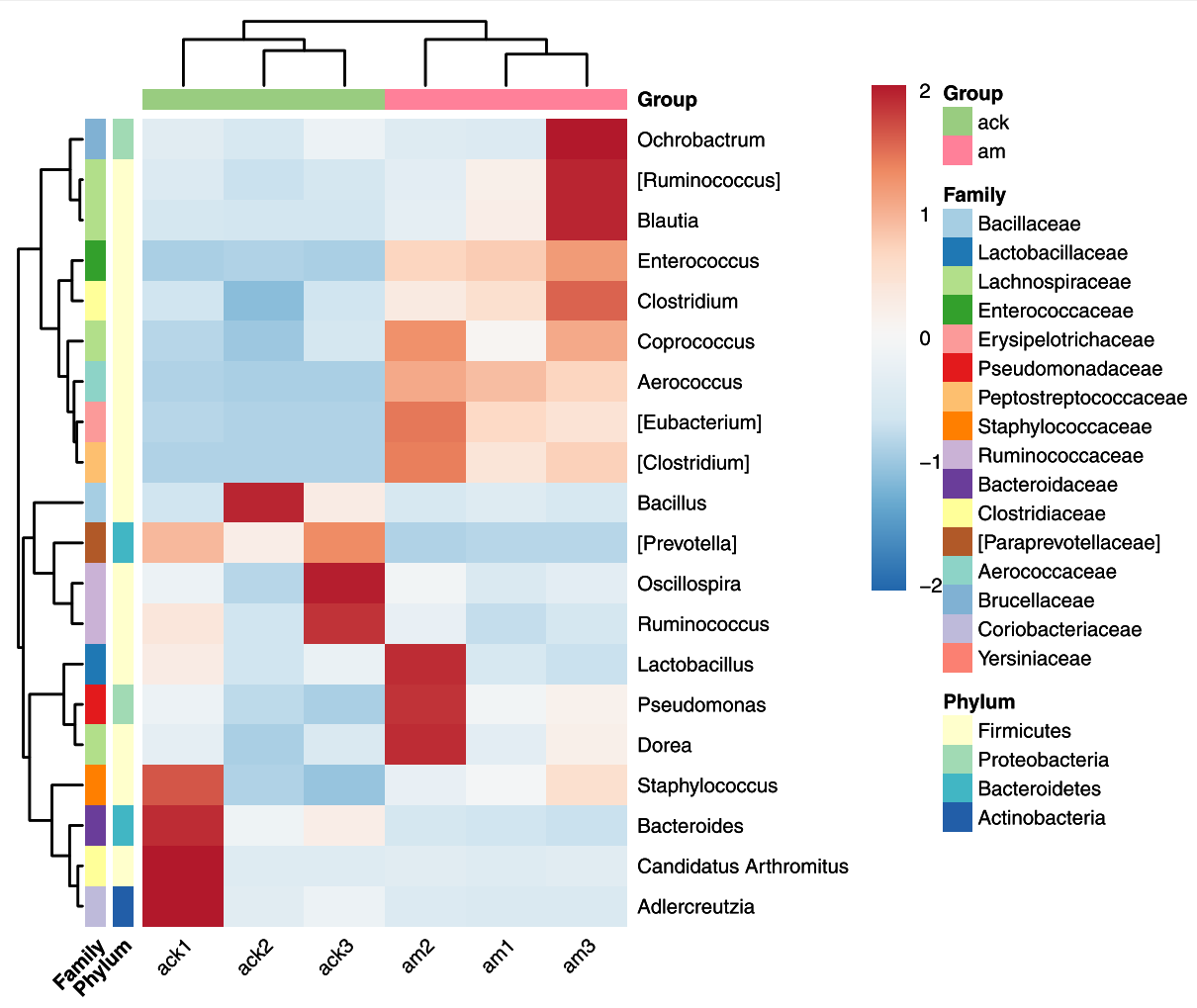

Fig. 6 Heat map of top 20 genera in abundance. The darker the red color, the higher the relative abundance is. The darker the blue color, the lower the relative abundance is. ack: control group, am: model group

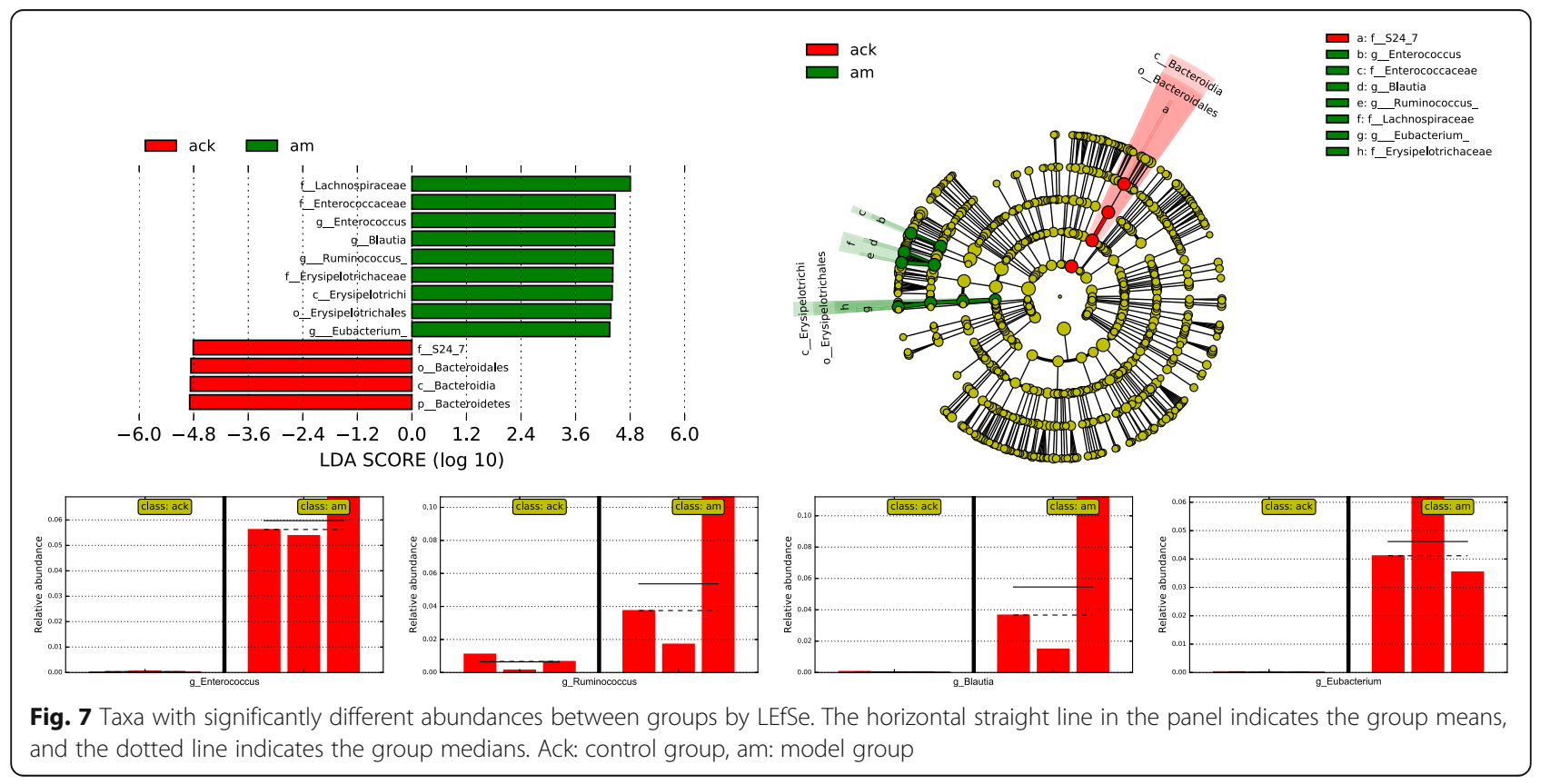


were possibly related to the occurrence of AAD in model mice.

\section{Discussion}

Changes in intestinal bacterial composition caused by antibiotics vary from antibiotic to antibiotic. Gentamicin is a broad-spectrum aminoglycoside with strong antibacterial activity against gram-negative bacteria. Cefradine, a broad-spectrum cephalosporin belongs to $\beta$-lactam, has a bactericidal effect on both gram-positive and gram-negative bacteria. In the previous study, we found the numbers of bacteria and colibacillus decreased rapidly with the use of gentamicin and cefradine. The number of yeast and mold presented an increasing trend [20]. Similar changes were found in other AAD models. Theriot et al. [21] treated mice with a variety of antibiotics to create distinct microbial and metabolic (bile acid) environments and found that susceptibility to $C$. difficile in the large intestine was observed only after specific broad-spectrum antibiotic treatment (cefoperazone, clindamycin, and vancomycin). These changes were correlated to the loss of members of families Lachnospiraceae and Ruminococcaceae [21]. Larcombe et al. [22] established an S. aureus infection model in mice pre-treated with kanamycin, gentamicin, colistin, metronidazole, vancomycin, and cefaclor. The results showed that colonization of various $S$. aureus strains could be achieved after antibiotic pre-treated [22]. Antibiotics lead to an alteration in bacteria composition resulting in changed metabolism and diminished anti-colonization.

The results, as direct evidence, suggested that the mice developed AAD was associated with alteration of the normal gut microbiota, which was mainly manifested as fewer beneficial bacteria and more potential pathogens. As shown in Fig. 3, after treatment with antibiotics, the number of bacterial species in intestinal contents decreased to different degrees at the levels of phylum, class, order, family, and genus. This result indicated that antibiotics changed the structure and density of normal intestinal flora, resulting in the disorder of intestinal flora. Specifically, to the level of phylum, the gut microbiota of normal mice was comprised of three dominant phyla, namely Firmicutes (63.52\%), Bacteroidetes (17.27\%), and Proteobacteria (13.07\%). As for AAD mice, it was transformed into Firmicutes (52.63\%) and Proteobacteria (46.37\%). There was a significantly higher F/B ratio in AAD mice. Similar shifts occurred in the intestinal mucosal bacteria of AAD mice [23]. Massive data identified Proteobacteria as a possible microbial signature of diseases which are sustained by various degrees of inflammation [24]. Notably, inflammation is demonstrated to be implicated in the development of metabolic disorders [25]. Thus, an increased abundance of Proteobacteria implies the risk of infection and metabolic disorder in a pathological state.

Furthermore, significant alterations in the gut microbiota composition were found at the genus levels. The dominant genera of healthy mice were Bacillus and Lactobacillus, which were beneficial to maintaining healthy intestinal flora and reducing the colonization of pathogenic organisms [26, 27]. However, with the administration of antibiotics, bacteria that were sensitive to gentamicin and/or cefradine were suppressed or killed, and bacteria that were resistant to them have the opportunity to invade and multiply. As a consequence, Ruminococcus, Blautia, Enterococcus, Eubacterium, Clostridium, Coprococcus, and Aerococcus were enriched in AAD mice (Fig. 6). Among these bacteria, the changes of Enterococcus and Eubacterium were especially prominent (Figs. 6, 7). In the AAD mice, the abundance of Enterococcus and Clostridium showed a significant increase, which was similar to the changes in the intestinal mucosa of AAD mice [23]. In our previous studies, we found a significant reduction in Lactobacillus in the intestinal mucosa of AAD mice, and the main genera in the intestinal mucosa of AAD mice were Enterococcus, Stenotrophomonas, Glutamicibacter, Citrobacter, and Pseudomonas [23]. The main genera in the intestinal contents of the same AAD model mice were Lactobacillus, Enterococcus, Blautia, [Ruminococcus], and Bacillus. Previous studies also showed that the role of intestinal microbiota in the development of AAD from the perspective of intestinal microbial function enzyme (lactase) gene. The main lactase-producing strains differed in the intestinal content and mucosa. The main lactaseproducing strain in the intestinal contents is Pseudomonas fluorescens [28], while the main lactase-producing strain in the intestinal mucosa is Stenotrophomonas [29]. Besides, antibiotics reduced the diversity of bacterial lactase genes in the intestinal contents but increased it in the intestinal mucosa [28, 29]. These dissimilarities in the composition and function of intestinal mucosal microbiota and intestinal contents microbiota provide new evidence for the spatial heterogeneity along the cross-section of the digestive tract (from lumen to mucosa). Factors known to drive this spatial heterogeneity along the longitudinal and transverse axes include chemical gradients (e.g., $\mathrm{pH}$ ), oxygen levels, nutrient availability, immune effectors, and functional heterogeneity of each gastrointestinal tract segment $[12,30]$.

Enterococcus are important opportunistic pathogens, with E. faecalis and E. faecium as the most representative species, causing a wide variety of infections. Many Enterococci have plasmid-encoded resistance genes which cause less susceptible to several antimicrobial agents intrinsically including gentamicin and cefradine 
[31-33]. Biofilm formation has been identified as an essential factor in the evasion of the host's immune response, the inhibitory or killing effects of antibiotics, and the pathogenesis of enterococcal infections [32, 34]. In addition, Enterococci are recognized as possessing a variety of virulence factors, which contribute to the mediation of adhesion, colonization, and invasion into the host tissues, modulation of the host immunity, and extra-cellular production of enzymes and toxins [35]. In this study, Enterococcus exhibited its intrinsic resistance to gentamicin and cefradine. Instead of being inhibited or killed, they proliferated in large quantities. A comparative genomic analysis discovered that the coregenome of Enterococcus obtains many genes related to carbohydrate metabolism and mannose, fructose, lactose, and galactose were the principal energy sources of $E n$ terococcus [36]. Based on previous studies and the results presented here, we propose that the overgrown Enterococcus may cause colonic infection and homeostasis disorder by forming a biofilm, possessing virulence factors, and adjusting carbohydrate metabolism. The specific mechanism needs further verification.

Another noteworthy bacterium was Clostridium. As known to all, $C$. difficile and $C$. perfringens are high-risk infectious origins of AAD. The former can produce an enterotoxin (toxin A) and a cytotoxin (toxin B), which cause mucosal injury and colonic inflammation [7]. The later can produce potent protein toxins ( $\alpha$-toxin, $\beta$-toxin, $\varepsilon$ toxin, and -toxin), which cause many different histotoxic and enterotoxic diseases in humans and animals [37]. There was no direct taxonomic evidence for $C$. difficile and C. perfringens in our data, but a significantly increased abundance of Clostridium also attracted our attention. We blasted the original sequence pairs that were classified into the genus Clostridium to the NCBI database separately. Then, a suspected strain of $C$. difficile and a suspected strain of $C$. perfringens were found. However, the identity of the specific strain remains to be further confirmed.

\section{Conclusions}

In summary, the bacterial diversity of the intestinal lumen was diminished after gentamicin and cefradine administration. The alterations in the abundance and composition of gut microbiota further led to the dysfunction of gut microbiota. More specifically, gentamicin and cefradine significantly increased the abundance of the opportunistic pathogens, of which Enterococcus and Clostridium are the most prominent and most worthy of attention.

\section{Methods}

\section{Experiment animals}

Six male and six female one-month-old specific pathogen free Kunming mice, weighing about $20 \pm 2 \mathrm{~g}$, were purchased from Hunan SJA Laboratory Animal Co., Ltd. (SCXK (Xiang) 2013-0004). These mice were housed under stable conditions (temperature $23-25^{\circ} \mathrm{C}$, relative humidity $50-70 \%, 12 \mathrm{~h}$ light/dark cycle) with unrestricted access to water and diet at the Experimental Animal Center of Hunan University of Chinese Medicine. The process of all animal experiments was conducted under animal protocols approved by the Institutional Animal Care and Use Committee of Hunan University of Chinese Medicine (Number: 20171202).

\section{Experiment reagents}

Gentamycin Sulfate Injection $(2 \mathrm{~mL})$ and Cefradine Capsules $(0.25 \mathrm{~g})$ were obtained from Yichang Renfu Pharmaceutical Co., Ltd. (Batch No.: 5120106) and Shanxi C\&Y Pharmaceutical Group Co., Ltd. (Batch No.: 110804). The mixture of antibiotics, at a concentration of $62.5 \mathrm{~g} \cdot \mathrm{L}^{-1}$, was prepared by Gentamicin sulfate injection, cefradine capsules, and physiological saline, and then reserved at $4{ }^{\circ} \mathrm{C}$.

\section{Animal experimental process and sample collection}

After 2 days of adaptive feeding, twelve mice were randomly divided into the control group (ack, 3 male and 3 female) and the model group (am, 3 male and 3 female). Then, female mice and male mice in the same group were fed separately housing (three male or three female mice per cage). The model group mice were administrated with the antibiotics mixture $0.35 \mathrm{~mL}$ per time by gavage, twice a day for 5 days $[3,20]$. Meanwhile, mice in the control group were given sterile saline with equal dose and frequency. The criteria of the AAD model are: (1)Frequency of defecation increased (2-3 times per day); (2)Feces becomes thin and soft; (3)Anus becomes dirty. Twelve mice were sacrificed by cervical dislocation on the 8th day, and their intestinal contents in the colon were collected separately under sterile conditions. To control the difference induced by gender, the intestinal contents of one male and one female from the same group were mixed. Then, six samples were loaded into EP tubes separately and frozen at minus $80^{\circ} \mathrm{C}$.

\section{PCR amplification and sequencing}

Metagenomic DNA was extracted from 6 samples using a modified cetyltrimethylammonium bromide (CTAB) method according to our previous reports $[28,38]$. The concentration and purity were measured by an Ultraviolet Spectrophotometer. Then, according to the concentration test results, the integrity of the DNA sample was detected by $0.8 \%$ agarose gel electrophoresis. The metagenomic DNA was diluted and used as a template. Then, combined with bacterial $16 \mathrm{~S}$ rDNA V4 region universal primer pair (520F: 5'-AYTGGGYDTAAAGNG-3' and 802R: 5'TACNVGGGTATCTAATCC-3'), the metagenomic DNA 
was amplified by PCR. After detected by $2 \%$ agarose gel electrophoresis, the PCR products were sequenced using the Illumina Miseq platform. PCR amplification and highthroughput sequencing were performed by Shanghai Personal Biotechnology Co., Ltd.

\section{Bioinformatics analysis}

Raw pair-end sequences were further filtered using Qiime software (version 1.7.0, http://qiime.org/) and removed chimeras using Mothur software (version 1.31.2, http:// www.mothur.org/) to obtain high-quality sequences which were finally used for subsequent analysis. Then, highquality sequences were clustered into operational taxonomic units (OTUs) with $97 \%$ similarity (3\% cutoff) using Qiime software, and chimeric sequences were identified and removed. Greengene database (Release $13.8 \mathrm{http}: / /$ greengenes.secondgenome.com/) was used to assigning taxonomy to the OTU table by the blast method in Qiime platform. After quality-filtering, a modified OTUs table was used for further analyses. The Venn diagram between groups was generated using $\mathrm{R}$ software. Four common diversity indices were calculated using Mothur software and visualized using GraphPad software. Based on the information of abundance at the genus level, the community structure map was drawn using MetaPhaAn software. LEfSe analysis was implemented to find biomarkers between two groups using the online tool hosted on the Galaxy web application [19] (https://huttenhower.sph.harvard.edu/galaxy/). LEfSe determines the features (operational taxonomic units, or taxa) most likely to explain differences between classes by coupling standard tests for statistical significance with additional tests encoding biological consistency and effect relevance [19]. A file compatible with LEfSe was created using the table of relative abundance and uploaded to the web application on Galaxy to run the LEfSe analysis. $p$-value of $<0.05$ was considered significant for statistical methods. Then, taxa with a logarithmic LDA score of over 4.0 were defined as biomarkers whose abundances were significantly increased compared to the other group.

\section{Statistical analysis}

When appropriate, data were presented as mean \& standard deviation $(\bar{x} \pm \mathrm{SD})$. All statistical analyses were performed in $\mathrm{R}$ (version 4.0.2), and raw $p$-value $<0.05$ and false discovery rate adjusted $p(p \mathrm{FDR})<0.05$ were considered as statistically significant. Mann-Whitney U test (non-parametric) was utilized to evaluate differences in characteristics of the normal group and model group. $p$-values were adjusted by Benjamini \& Hochberg (BH) correction.

\section{Abbreviations}

AAD: Antibiotic-associated diarrhea; ADE: Adverse drug event:

OTU: Operational taxonomic unit; CTAB : Cetyltrimethylammonium bromide;
PCoA: Principal coordinates analysis; LDA: Linear discriminant analysis; LEfSe: Linear discriminant analysis effect size; F/B : Firmicutes to Bacteroidetes ratio

\section{Acknowledgements}

Thanks for the sequencing service provided by Personal Biotechnology Co. Ltd.

\section{Authors' contributions}

$\mathrm{ZT}$ designed the research. HS and ZC supervised the research work and guided the experimental design. NX provided the suggestion of the research work. HS prepared the manuscript. All authors contributed to interpretation of the results and manuscript drafting. All authors read and approved the final manuscript.

\section{Funding}

This work was supported by grants from the National Natural Science Foundation of China (No: 81573951). The author of this paper was in charge of the project, and Professor Zhoujin Tan is the project leader. Funding body provided approval for the manuscript and had no role in design of the study, analysis and interpretation of data.

Availability of data and materials

The datasets used and analyzed during the current study are available from the corresponding author on reasonable request.

Ethics approval and consent to participate

SPF-grade KM mice were purchased from Hunan SJA Laboratory Animal Co., Ltd. (SCXK (Xiang) 2013-0004). All animal work was carried out following accordance within the guidelines of the Institutional Animal Care and Use Committee of Hunan University of Chinese Medicine (NO.20171202).

\section{Consent for publication}

Not applicable.

\section{Competing interests}

The authors have no conflict of interest to declare.

\section{Author details}

${ }^{1}$ School of Traditional Chinese Medicine, Hunan University of Chinese Medicine, Changsha, Hunan, China. ${ }^{2}$ Hunan Key Laboratory of TCM Prescription and Syndromes Translational Medicine, Changsha, Hunan, China. ${ }^{3}$ School of Pharmaceutical Science, Hunan University of Chinese Medicine, Changsha, Hunan, China. ${ }^{4}$ School of Medicine, Hunan University of Chinese Medicine, Changsha, Hunan, China.

Received: 2 June 2020 Accepted: 7 October 2020

Published online: 15 October 2020

\section{References}

1. Bartlett JG. Clinical practice. Antibiotic-associated diarrhea. N Engl J Med. 2002;346(5):334-9. https://doi.org/10.1056/NEJMcp011603.

2. Mantegazza C, Molinari P, D'Auria E, Sonnino M, Morelli L, Zuccotti GV. Probiotics and antibiotic-associated diarrhea in children: a review and new evidence on Lactobacillus rhamnosus GG during and after antibiotic treatment. Pharmacol Res. 2018;128:63-72. https://doi.org/10.1016/j.phrs. 2017.08.001.

3. Zeng A, Zhang H, Tan Z, Cai Y, Cai G, Zhou S. The construction of mice diarrhea model due to dysbacteriosis and curative effect of ultra-micro Qiweibaizhusan. Microbiol China. 2012;39(9):1341-8. https://doi.org/10. 13344/j.microbiol.china.2012.09.012.

4. Clausen MR, Bonnén H, Tvede M, Brøbech MP. Colonic fermentation to short-chain fatty acids is decreased in antibiotic-associated diarrhea. Gastroenterology. 1991;101(6):1497-504. https://doi.org/10.1016/00165085(91)90384-W.

5. Silverman MA, Konnikova L, Gerber JS. Impact of antibiotics on necrotizing Enterocolitis and antibiotic-associated diarrhea. Gastroenterol Clin N Am. 2017:46(1):61-76. https://doi.org/10.1016/j.gtc.2016.09.010.

6. Hogenauer C, Hammer HF, Krejs GJ, Reisinger EC. Mechanisms and management of antibiotic-associated diarrhea. Clin Infect Dis. 1998;27(4): 702-10. https://doi.org/10.1086/514958. 
7. Guery B, Galperine T, Barbut F. Clostridioides difficile: diagnosis and treatments. BMJ. 2019;366:14609. https://doi.org/10.1136/bmj.|4609.

8. Larcombe S, Hutton ML, Lyras D. Involvement of Bacteria other than Clostridium difficile in antibiotic-associated Diarrhoea. Trends Microbiol. 2016;24(6):463-76. https://doi.org/10.1016/j.tim.2016.02.001.

9. Lozupone CA, Stombaugh II, Gordon JI, Jansson JK, Knight R. Diversity, stability and resilience of the human gut microbiota. Nature. 2012;489(7415): 220-30. https://doi.org/10.1038/nature11550.

10. Ma Q, Li Y, Li P, Wang M, Wang J, Tang Z, et al. Research progress in the relationship between type 2 diabetes mellitus and intestinal flora. Biomed Pharmacother. 2019;117:109138. https://doi.org/10.1016/j.biopha.2019.109138.

11. Greenhalgh K, Meyer KM, Aagaard KM, Wilmes P. The human gut microbiome in health: establishment and resilience of microbiota over a lifetime. Environ Microbiol. 2016;18(7):2103-16. https://doi.org/10.1111/14622920.13318.

12. Martinez-Guryn $K$, Leone V, Chang EB. Regional diversity of the gastrointestinal microbiome. Cell Host Microbe. 2019;26(3):314-24. https:// doi.org/10.1016/j.chom.2019.08.011.

13. He Y, Wen Q, Yao F, Xu D, Huang Y, Wang J. Gut-lung axis: the microbial contributions and clinical implications. Crit Rev Microbiol. 2017;43(1):81-95 https://doi.org/10.1080/1040841X.2016.1176988.

14. Sittipo P, Shim JW, Lee YK. Microbial Metabolites Determine Host Health and the Status of Some Diseases. Int J Mol Sci. 2019;20(21). https://doi.org/ 10.3390/ijms20215296.

15. Cryan JF, O'Riordan KJ, Cowan CSM, Sandhu KV, Bastiaanssen TFS, Boehme M, et al. The microbiota-gut-brain Axis. Physiol Rev. 2019;99(4):1877-2013. https://doi.org/10.1152/physrev.00018.2018.

16. Lynch SV, Pedersen O. The human intestinal microbiome in health and disease. N Engl J Med. 2016;375(24):2369-79. https://doi.org/10.1056/ NEJMra1600266.

17. Juul FE, Garborg K, Bretthauer M, Skudal H, Oines MN, Wiig H, et al. Fecal microbiota transplantation for primary Clostridium difficile infection. N Engl J Med. 2018;378(26):2535-6. https://doi.org/10.1056/NEJMc1803103.

18. Guo Q, Goldenberg JZ, Humphrey C, El Dib R, Johnston BC. Probiotics for the prevention of pediatric antibiotic-associated diarrhea. Cochrane Database Syst Rev. 2019;4:CD004827. https://doi.org/10.1002/14651858. CD004827.pub5

19. Segata N, Izard J, Waldron L, Gevers D, Miropolsky L, Garrett WS, et al. Metagenomic biomarker discovery and explanation. Genome Biol. 2011; 12(6):R60. https://doi.org/10.1186/gb-2011-12-6-r60.

20. Tan Z, Zhang H, Zhou S, Yu W, Zeng A, Cai Y, et al. R285.5Change of intestinal microbes in Dysbacteriosis-modeled mice treated with ultra-micro powder of Qiweibaizhusan. Chin J Appl Environ Biol. 2013;19(3):449-53. https://doi.org/10.3724/SP.J.1145.2013.00449.

21. Theriot CM, Bowman AA, Young VB. Antibiotic-Induced Alterations of the Gut Microbiota Alter Secondary Bile Acid Production and Allow for Clostridium difficile Spore Germination and Outgrowth in the Large Intestine. mSphere. 2016;1(1). https://doi.org/10.1128/mSphere.00045-15.

22. Larcombe S, Jiang JH, Hutton ML, Abud HE, Peleg AY, Lyras D. A mouse model of Staphylococcus aureus small intestinal infection. J Med Microbiol. 2020;69(2):290-7. https://doi.org/10.1099/jmm.0.001163.

23. Xie G, Tan K, Peng M, Long C, Li D, Tan Z. Bacterial diversity in intestinal mucosa of antibiotic-associated diarrhea mice. 3 Biotech. 2019;9(12):444. https://doi.org/10.1007/s13205-019-1967-2.

24. Shin NR, Whon TW, Bae JW. Proteobacteria: microbial signature of dysbiosis in gut microbiota. Trends Biotechnol. 2015;33(9):496-503. https://doi.org/10. 1016/j.tibtech.2015.06.011.

25. Rizzatti G, Lopetuso LR, Gibiino G, Binda C, Gasbarrini A. Proteobacteria: a common factor in human diseases. Biomed Res Int. 2017;2017:9351507. https://doi.org/10.1155/2017/9351507.

26. Wu H, Xie S, Miao J, Li Y, Wang Z, Wang M, et al. Lactobacillus reuteri maintains intestinal epithelial regeneration and repairs damaged intestinal mucosa. Gut Microbes. 2020;11(4):997-1014. https://doi.org/10.1080/ 19490976.2020.1734423.

27. Piewngam $P$, Zheng $Y$, Nguyen TH, Dickey SW, Joo HS, Villaruz AE, et al. Pathogen elimination by probiotic Bacillus via signalling interference. Nature. 2018;562(7728):532-7. https://doi.org/10.1038/s41586-018-0616-y.

28. Long CX, He L, Guo YF, Liu YW, Xiao NQ, Tan ZJ. Diversity of bacterial lactase genes in intestinal contents of mice with antibiotics-induced diarrhea. World J Gastroenterol. 2017;23(42):7584-93. https://doi.org/10. 3748/wjg.v23.i42.7584.
29. Long C, Liu Y, He L, Tan Q, Yu Z, Xiao N, et al. Bacterial lactase genes diversity in intestinal mucosa of mice with dysbacterial diarrhea induced by antibiotics. 3 Biotech. 2018;8(3):176. https://doi.org/10.1007/ s13205-018-1191-5.

30. Tropini C, Earle KA, Huang KC, Sonnenburg JL. The gut microbiome: connecting spatial organization to function. Cell Host Microbe. 2017;21(4): 433-42. https://doi.org/10.1016/j.chom.2017.03.010.

31. Chow JW. Aminoglycoside resistance in enterococci. Clin Infect Dis. 2000; 31(2):586-9. https://doi.org/10.1086/313949.

32. Weng PL, Ramli R, Hamat RA. Antibiotic Susceptibility Patterns, Biofilm Formation and esp Gene among Clinical Enterococci: Is There Any Association? Int J Environ Res Public Health. 2019;16(18). https://doi.org/10. 3390/ijerph16183439.

33. Torres C, Alonso CA, Ruiz-Ripa L, Leon-Sampedro R, Del Campo R, Coque TM. Antimicrobial Resistance in Enterococcus spp. of animal origin. Microbiol Spectr. 2018;6(4). https://doi.org/10.1128/microbiolspec.ARBA0032-2018.

34. Heikens E, Bonten MJ, Willems RJ. Enterococcal surface protein Esp is important for biofilm formation of Enterococcus faecium E1162. J Bacteriol. 2007;189(22):8233-40. https://doi.org/10.1128/JB.01205-07.

35. Strateva T, Atanasova D, Savov E, Petrova G, Mitov I. Incidence of virulence determinants in clinical Enterococcus faecalis and Enterococcus faecium isolates collected in Bulgaria. Braz J Infect Dis. 2016;20(2):127-33. https://doi. org/10.1016/j.bjid.2015.11.011.

36. Zhong Z, Zhang W, Song Y, Liu W, Xu H, Xi X, et al. Comparative genomic analysis of the genus Enterococcus. Microbiol Res. 2017;196:95-105. https:// doi.org/10.1016/j.micres.2016.12.009.

37. Rood Jl, Adams V, Lacey J, Lyras D, McClane BA, Melville SB, et al. Expansion of the Clostridium perfringens toxin-based typing scheme. Anaerobe. 2018; 53:5-10. https://doi.org/10.1016/j.anaerobe.2018.04.011.

38. Wu H, Zhou S, Guo C, Tan Z, Cai G, Zeng A, et al. A metagenome DNA extracting method of intestinal flora in mice for molecular diversity analysis based on PCR technology. Chin J Microecol. 2012;24(7):648-51. https://doi. org/10.13381/j.cnki.cjm.2012.07.003.

\section{Publisher's Note}

Springer Nature remains neutral with regard to jurisdictional claims in published maps and institutional affiliations.
Ready to submit your research? Choose BMC and benefit from:

- fast, convenient online submission

- thorough peer review by experienced researchers in your field

- rapid publication on acceptance

- support for research data, including large and complex data types

- gold Open Access which fosters wider collaboration and increased citations

- maximum visibility for your research: over $100 \mathrm{M}$ website views per year

At BMC, research is always in progress.

Learn more biomedcentral.com/submissions 